\title{
The Quality of Lifestyle and the Quality of Life
}

\author{
Militza Moreno, MD [Co-Author] \\ Postdoctoral Fellow in Preventive Cardiology Department of Community and Preventive Medicine \\ University of Rochester School of Medicine and Dentistry Militza_moreno@urmc.rochester.edu
}

Thomas A. Pearson, MD, MPH, PhD [Co-Author] and Albert D. Kaiser [Professor] Department of Community and Preventive Medicine University of Rochester School of Medicine and Dentistry thomas_pearson@urmc.rochester.edu

Clinical and public health professionals committed to the prevention of heart disease and stroke have long had an "image" problem. Purveyors of goods and services now recognized as part of unhealthy lifestyles have long appealingly marketed themselves using terms like "zesty" (for high sodium content), "rich" (for high saturated fat), "satisfying" for cigarettes laden with tar and nicotine, etc. Various campaigns to improve the U.S. diet, increase physical activity, reduce exposure to tobacco smoke, and other healthy lifestyles have been met with endless jokes and cartoons, lampooning efforts to reduce risky lifestyle behaviors as an attack on an admirable quality of life. At the same time, the preventive cardiology community may not have been optimally effective in a campaign of fear and loathing in conveying the contributions of heart disease and stroke to a very poor quality of life, as motivation to adopt healthy lifestyles and, if necessary, to adhere to treatment regimes to reduce cardiovascular risk.

In this volume of the Archives, two papers add to this discussion, as both link unhealthy lifestyles with conditions well recognized to confer a poor quality of life. Y. Zhang and colleagues from China, U.S., and Finland have examined the relationship between five lifestyle indicators (smoking, body mass index, physical activity, vegetable consumption, and alcohol consumption) in five cross-sectional population surveys performed across Finland between 1982 and 2002, involving 367,686 Finnish participants aged 25-74 years without coronary disease and stroke. Even after adjustment for demographic factors and reported histories of diabetes, hypertension, and elevated blood cholesterol, strong, inverse, and graded associations between the number of healthy lifestyles and the risks total, ischemic, and hemorrhagic stroke were identified. In a second paper from the Mayo Clinic, Gupta, et al. performed a systematic review and metaanalysis of interventions on lifestyles or risk-reducing pharmacotherapy on the severity of erectile dysfunction in men. Six clinical trials involving 740 participants demonstrated significant improvements in sexual function with lifestyle intervention alone or with treatment of risk factors (e.g. statins for hyperlipidemia).

These two reports describe meaningful benefits of healthy lifestyle on cerebrovascular disease and erectile dysfunction. They add to the rich data showing reduction of myocardial infarction and cardiac death in the absence of the risk behaviors $(1,2)$. Unfortunately, despite these persuasive benefits, cardiac risk factors are still rampant and increasing in Western societies (3). Indeed, in the paper by Zhang, et al., only $7.5 \%$ of the Finnish population had all five healthy risk behaviors.

Corresponding Author: Thomas A. Pearson, MD, MPH, PhD University of Rochester Medical Center 265 Crittenden Boulevard, CU 420708 Rochester, New York 14642-0708 Tel: 585-275-0663 Fax: 585-276-1122 thomas_pearson@urmc.rochester.edu. 
The number of health benefits that accrue to persons with healthy lifestyles continue to increase in number and importance. Many Americans seek treatment, often pharmacologic for erectile dysfunction (ED), which may result from perturbations in vascular, neurological, psychological, and other factors. ED is known to be related to the established risk factors from coronary disease (hypertension, hyperlipidemia, obesity, and diabetes) (4) and could be considered a cardiovascular risk marker $(5,6)$. Just the ability of a healthier lifestyle to reduce ED severity (or prevent its occurrence) should be a strong and persuasive argument for many. Even after adjusting for other physiological risk factors (hypertension, diabetes, hyperlipidemia) Zhang, et al. still identified a beneficial role for healthy lifestyles. Finally, healthy lifestyles also potentiates pharmacotherapy, such as a low saturated fat/cholesterol diet improving control of hyperlipidemia with cholesterol-lowering drugs, and diet and exercise are the foundation of any therapy for Type II diabetes.

Ischemic stroke, hemorrhagic stroke, and erectile dysfunction can be added to the growing list of lifestyle-related diseases. Another notable addition to this list is congestive heart failure (7). These newly observed associations are meaningful, in that these diseases are significant detractors to quality of life. The sequelae of nonfatal strokes are the leading cause of admission to nursing homes in the U.S. (8). Congestive heart failure is the leading cause of most admissions to inpatient medical wards (9). Erectile dysfunction is identified as one of the main causes of impaired quality of life in the U.S., affecting millions of men (10).

The increasing epidemic of obesity in the US is a clarion call to step up our efforts to motivate our patients and the public-at-large to make even small changes towards healthier lifestyles. These new associations between healthy lifestyles and reducing incidence of stroke, congestive heart failure, and erectile dysfunction can add additional powerful persuaders. While recently introduced cigarette packaging with graphic reminders of the truly unpleasant consequences of cigarette smoking, referred to as "terror campaigns", in the modification of patient behaviors remains controversial, two points should be emphasized. First, our patients have the right to be informed of the consequences of their unhealthy behaviors. Stroke, heart failure, and erectile dysfunction expand the list of conditions which are highly desirable to avoid. Second, for healthcare providers who provide advice and counseling, the information may be used selectively in the patient with a family member or friend who has suffered from a disabling stroke, or the male patient now beginning treatment for ED. While the greatest benefit in sheer numbers is the prevention of coronary disease, it may be the motivation to prevent one of these other poor outcomes that tips the balance toward lifestyle change. Finally, for the health system and third party payers, reimbursement for the time and effort of healthcare professionals to provide counseling on lifestyle change or referral to services that provide these services remains inconsistent and frequently unsupported. The cost of admissions, physician visits, and pharmacotherapy for stroke and erectile dysfunction are certainly enormous. Both clinicians and public health practitioners should be reassured that the benefits of their lifestyle modification efforts are overwhelmingly positive and continue to grow, and we should renew our efforts to help patients add life to the years, as well as years to life.

\section{Acknowledgments}

Supported in part by an Institutional Research Career Development Award (HL-T32-HL007937) from the National Heart, Lung and Blood Institute, NIH

\section{References}

1. Stamler J, Stamler R, Neaton JD, et al. Low risk-factor profile and long-term cardiovascular and noncardiovascular mortality and life expectancy: findings for 5 large cohorts of young adult and middle-aged men and women. JAMA. Dec 1; 1999 282(21):2012-2018. [PubMed: 10591383] 
2. Stampfer, et al. Primary Prevention of Coronary Heart Disease in Women through Diet and Lifestyle. NEJM. 2000; 343:16-22. [PubMed: 10882764]

3. Lloyd-Jones DM, et al. Prediction of Lifetime Risk for Cardiovascular Disease by Risk Factor Burden at 50 Years of Age. Circulation. 2006; 113:791-798. [PubMed: 16461820]

4. Lue TF. Erectile Dysfunction. NEJM. 2000; 342:1802-1803. [PubMed: 10853004]

5. Potenza MA, Gagliardi S, Nacci C, et al. Endothelial dysfunction in diabetes: from mechanisms to therapeutic targets. Curr Med Chem. 2009; 16:94-112. [PubMed: 19149564]

6. Khan MA, Ledda A, Mikhailidis DP, et al. for Consensus Writing Committee. Second Consensus Conference on Cardiovascular Risk Factors and Erectile Dysfunction. Curr Med Res Opin. 2002; 18:33-35. [PubMed: 11999144]

7. Djoussé L, et al. Between Modifiable Lifestyle Factors and Lifetime Risk of Heart Failure. JAMA. 2009; 302(4):394-400. [PubMed: 19622818]

8. Roger VL, et al. Heart Disease and Stroke Statistics--2011 Update: A Report from the American Heart Association. Circulation. 2011; 123:e18-e209. [PubMed: 21160056]

9. Liu L. Changes in Cardiovascular Hospitalization and Comorbidity of Heart Failure in the United States: Findings from the National Hospital Discharge Surveys 1980-2006. International Journal of Cardiology. 2011; 49:39-45. [PubMed: 20060181]

10. Laumann EO, Paik A, Rosen RC. Sexual dysfunction on the United States: prevalence and predictors. JAMA. 1999; 281:537-544. [PubMed: 10022110] 